\title{
CRONICIDAD Y CUIDADORES FAMILIARES: UNA REVISIÓN DESDE LO CONTEXTUAL Y CONCEPTUAL
}

Por:

Olga Marina Vega Angarita*, Dianne Sofia Gonzalez Escobar**, Ma. Mercedes Ramirez Ordoñez.***

\section{RESUMEN}

Este artículo se genera a partir de un estudio multicéntrico sobre la implementación y evaluación del Programa Cuidando a los Cuidadores desarrollado con la participación de nueve países de Latinoamérica y unidades académicas de enfermería en Colombia entre ellas la Universidad Francisco de Paula Santander de Cúcuta, en coordinación con la universidad Nacional de Colombia. El artículo presenta inicialmente la contextualización de lo sociodemográfico y epidemiológico del envejecimiento y de las enfermedades crónicas no transmisible a nivel mundial, de Latinoamérica y en el contexto regional. Seguidamente se abordan desde lo conceptual los cuidadores familiares como directos protagonistas de la llamada Red del cuidado informal; finalmente se analizan las repercusiones del cuidado de enfermos crónicos sobre las diferentes áreas de la vida y según datos de estudios previos.

PALABRAS CLAVE: Enfermedades crónicas, cuidadores familiares, repercusiones del cuidado.

\section{ABSTRACT}

The article is generated from a multicentric study on the implementation and evaluation of the program taking care of to the caretakers, developed at the moment by the National University of Colombia with the participation nine countries of Latin America and Academic units of infirmary in Colombia among them the University Francisco de Paula Santander. The article presents/displays initially the contextualization from the sociodemográfic and epidemiologist of the aging and the chronic diseases nontransmissible at worldwide level, of Latin America and in the regional context. Next they approach from conceptual the familiar caretakers like direct protagonists of the call network of the informal care, finally one analyzes the repercussions of the care of chronic patients on the different areas from the life and according to data of previous studies.

Keywords: Chronic diseases, Caregivers, repercussions of the care.

\section{ENFERMEDAD CRÓNICA: DIMENSIONES SOCIODEMOGRÁFICA Y EPIDEMIOLÓGICA}

Durante el siglo XX la tasa de crecimiento demográfico ha presentado cambios significativos en el mundo y en los países de América Latina. La tendencia existente es la de mayor aumento de la población en los países más subdesarrollados de la región. En Estados Unidos y Canadá se registra una disminución del $40 \%$ en el crecimiento demográfico; estas tasas varían desde la más baja 1,06\%, en Estados Unidos y Canadá, pasando por valores intermedios del 2,75\% para los países andinos, hasta un valor extremo $4,1 \%$ en Venezuela'.

Así, los adelantos tecnológicos y científicos han traído

\footnotetext{
*Enfermera Magíster en Enfermería con énfasis en cuidado al paciente crónico, Especialista Medicoquirúrgica con énfasis en neurocirugía. Docente Asociado Facultad Ciencias de la Salud, Universidad Francisco de Paula Santander, Grupo de investigación de cuidado de enfermería E-Mail: omvega@bari.ufps.edu.co.

** Docente Asociado Facultad Ciencias de la Salud, Universidad Francisco de Paula Santander, Enfermera Magíster en Enfermería con énfasis en cuidado al paciente crónico, Especialista Docencia Universitaria. Grupo de investigación de cuidado de enfermería E-Mail: dsgonzal@bari.ufps.edu.co.

Docente Asociado Facultad Ciencias de la Salud, Universidad Francisco de Paula Santander, Magíster en Enfermería con énfasis en cuidado al paciente crónico, Especialista en paciente renal, Especialista Docencia Universitaria.

1 OPS/OMS. La enfermería en la región de las Américas. Washington, 1999, p. 1.
} 


\section{CRONICIDAD Y CUIDADORES FAMILIARES: UNA REVISIÓN DESDE LO CONTEXTUAL Y CONCEPTUAL}

a la humanidad la disminución de las enfermedades de tipo transmisible, de tal forma la esperanza de vida ha aumentado de 3.3 a 5.7 años en América Latina entre 1955 y $2000^{2}$; sumado a las tendencias en el control en la natalidad la pirámide poblacional muestra un aumento en el número de adultos mayores.

El envejecimiento de la población muestra un aumento de las enfermedades crónicas y discapacitantes. Por lo general, las enfermedades diagnosticadas en los adultos mayores son incurables y, si no se tratan adecuada y oportunamente, tienden a provocar complicaciones y secuelas que dificultan la independencia y la autonomía de las personas. La salud no puede ser medida solo en términos de la presencia o ausencia de enfermedad, debe valorar también el grado de conservación de la capacidad funcional. La discapacidad acarrea el riesgo de morir, independientemente de la edad, el sexo y de la presencia de otras afecciones. A pesar de que la mayoría de las personas de edad con enfermedades no trasmisibles mantienen su capacidad funcional; el grado de discapacidad aumenta con la edad.

La Organización Mundial de la Salud, OMS, afirma que cerca del $60 \%$ de las muertes que ocurren en el mundo hoy se deben a las enfermedades crónicas, en especial la enfermedad cardiaca, la enfermedad cerebro vascular, el cáncer y la enfermedad pulmonar.

El 20\% de las muertes por enfermedades crónicas se producen en los países de altos ingresos, mientras que el $80 \%$ se registra en países de ingresos bajos en los cuales vive la mayor parte de la población y en donde cada vez es mayor el número de personas, familias y comunidades afectadas en su calidad y esperanza de vida $^{3}$.

La población colombiana se caracteriza por envejecimiento mientras las tasas de fecundidad y de natalidad van en decremento. La esperanza de vida al nacer se estima en 69,2 años para los hombres y 75.3 años para las mujeres, la disminución en la mortalidad infantil, en especial por enfermedades transmisibles ha incidido en la disminución de los años de vida potencial perdidos ${ }^{4}$.

El país vive una transición caracterizada por la mejora progresiva, pero desigual, y la concurrencia de enfermedades transmisibles, crónicas y degenerativas que afectan con mayor severidad a la población más pobre con diferencias evidentes de género. Se observa una morbimortalidad creciente asociada sobre todo a enfermedades relacionadas con malos hábitos alimenticios, el cigarrillo, consumo de bebidas alcohólicas y la falta de ejercicio. Así, la primera causa de muerte son las enfermedades cardiovasculares con 113,4 muertes, y la tercera causa los tumores malignos con 61,3 muertes por cada 100.000 habitantes $^{5}$.

En Norte de Santander la situación social y de salud es muy similar a la del resto del país como lo muestra el Boletín Epidemiológico del 20056 . El Departamento cuenta con una población para ambos sexos proyectado para el 2005 de 1.494 .219 habitantes. De los cuales 158.806 son personas mayores de 45 años.

2 ORGANIZACIÓN MUNDIAL DE LA SALUD. Prevención de las enfermedades crónicas una inversión vital. Nueva York: OMS, 2002, p. 23.

${ }^{3}$ ORGANIZACIÓN MUNDIAL DE LA SALUD. Prevención de las enfermedades crónicas: una inversión vital. Los países pobres los más afectados. s. I: OMS, 2006, 2 p.

${ }^{4}$ DANE, "Proyecciones departamentales de población por sexo y edad", 1990-2015.

${ }^{5}$ Ministerio de Salud y OPS. "Situación de salud en Colombia. Indicadores básicos 2001", Estándares (documento).

${ }^{6}$ Boletín Epidemiológico del Norte de Santander, vol. 6 del 2005. 


\section{CRONICIDAD Y CUIDADORES FAMILIARES: UNA REVISIÓN DESDE LO CONTEXTUAL Y CONCEPTUAL}

A nivel socioeconómico, el $93.45 \%$ de la población en el Departamento de ubica en el nivel 1 y 2, el estado de aseguramiento para el año 2004 es del 50.4. La morbilidad hospitalaria en el 2004 para las edades entre 45 y 59 años, según orden de aparición, se encuentra entre las 10 primeras causas: enfermedades mentales no especificadas, traumatismo intracraneal, diarrea y gastroenteritis de presunto origen infeccioso, trastornos de la piel y del tejido subcutáneo, infección de vías urinarias, asma no especificada, prolapso útero vaginal, fracturas de región no especificada del cuerpo, y la insuficiencia cardiaca congestiva. Para la población mayor de 60 años se destacan la insuficiencia cardiaca congestiva, traumatismos no especificados, hipertensión arterial, enfermedad cardiaca hipertensiva, fractura fémur y accidente cerebro vascular agudo.

Los reportes estadísticos de instituciones de la región con alto cubrimiento a pacientes en situación de cronicidad como la ESE Hospital Universitario Erasmo Meoz de Cúcuta, ratifican que las enfermedades crónicas no transmisibles representan un problema de salud pública. Cuantitativamente, dentro de las 10 primeras causas de admisión hospitalaria se encontró que las enfermedades cardiovasculares alcanzan el $44.7 \%$, los problemas respiratorios el $30.18 \%$ y las enfermedades renales el $4.71 \%$.

Identificada esta problemática, y al tener claridad en los cambios epidemiológicos presentados, las enfermedades crónicas se han convertido en la principal causa de mortalidad e incapacidad para la población adulta. Estas enfermedades ocasionan padecimientos de larga duración, de carácter no transmisible y degenerativo. Se caracterizan por una etiología incierta, múltiples factores de riesgo, períodos de latencia largos, curso prolongado, fuente no contagiosa, discapacidad o alteración funcional e incurabilidad.

Las enfermedades crónicas afectan a todos los grupos de la sociedad e imponen dos tipos de carga: de una parte, afectan la productividad de los individuos y su capacidad de generar ingresos y, de otra originan un mayor consumo de servicios sociales y de salud, por lo general de alto costo. Se aspira que para el 2020 todos los países hayan introducido y se encuentren manejando y evaluando estrategias que fortalezcan los estilos de vida saludables a través de una combinación de programas comunitarios, económicos, educativos, organizacionales y de regulación.

Se presume que en América Latina el tiempo, la dedicación y el cuidado informal es mayor debido a la situación económica de los países subdesarrollados en donde se cuenta con escasos sistemas de salud que no alcanzan a cubrir la totalidad de la población y, además, fallan en momentos determinados ofreciendo un servicio ineficaz.

En la actualidad existen programas que han dado su mirada a la situación de aumento de las enfermedades crónicas. La Organización Mundial de la Salud y la Organización Panamericana de la Salud, como el CARMEN (Conjunto de Acciones para la Intervención Multifactorial de Enfermedades no Transmisibles), son avances importantes en la organización social en este campo.

La finalidad del Proyecto Carmen es mejorar la salud de la población de un país mediante la reducción de

\footnotetext{
${ }^{7}$ Estadística ESE Hospital Universitario Erasmo Meoz, 50 primeras causas de morbilidad sentida en el servicio de medicina interna, año 2005.
} 


\section{CRONICIDAD Y CUIDADORES FAMILIARES: UNA REVISIÓN DESDE LO CONTEXTUAL Y CONCEPTUAL}

la incidencia de los factores de riesgo asociados a las enfermedades no transmisibles mediante acciones combinadas y coordinadas de promoción de la salud y prevención de la enfermedad.

En Norte de Santander existe en el momento un grupo de personas del orden gubernamental que desarrolla, a través de la Red Municipal, una serie de actividades para la prevención y control de las enfermedades crónicas, con los siguientes objetivos:

1. Integrar los esfuerzos públicos y privados para asesorar en el diseño y desarrollo de modelos de intervención comunitaria e intersectorial a nivel municipal basados en la protección de la salud, la detección temprana y el control de las enfermedades y factores de riesgo asociados.

\section{Fomentar el compromiso intersectorial,} interinstitucional y comunitario de todo el municipio para promover comportamientos, hábitos y estilos de vida saludables, y prevenir y controlar enfermedades y factores de riesgo de acuerdo con los perfiles epidemiológicos locales.

3. Diseñar estrategias, líneas de acción y formulación de políticas públicas que involucren a todos los estamentos públicos y privados que existen en el municipio, que busquen un mayor impacto de sus acciones en la comunidad.

4.Garantizar un manejo adecuado, oportuno y eficiente de todos los recursos humanos, físicos, técnicos, administrativos y financieros que posea la red.

5. Gestionar recursos internacionales, nacionales, departamentales, municipales, tanto públicos como privados, que permitan la consecución de los objetivos propuestos.

6. Consolidar un trabajo de equipo con todas las instituciones que conformen la Red Municipal, despertando el sentido de pertenencia y compromiso con las tareas propuestas para lograr un mayor impacto en la comunidad.

\section{CUIDADOR FAMILIAR: Conceptualización}

El bienestar de los enfermos crónicos depende, en gran medida, de las personas que conviven con ellos en el ámbito del cuidado informal. El Ilamado cuidado informal es aquél que se presta a personas dependientes (enfermos, discapacitados o ancianos) por familiares, amigos $y$, en general, personas de la Red Social inmediata, sin recibir retribución económica por la labor que se ofrece. Por lo general, de índole gratuita en razón de relaciones afectivas y de parentesco, y por efectuarse en el ámbito doméstico ${ }^{8}$.

Ofrecer una atención sin límites de tiempo, tener un elevado grado de compromiso hacia la tarea realizada y de sentido del deber, la falta de preparación para asumir el rol, son otros aspectos característicos que precisan su definición.

No existe un recurso más relevante para la salud que el cuidado informal. En forma cuantitativa supone más del $88 \%$ del total de los cuidados que se realizan. Múltiples estudios ponen de manifiesto la magnitud e importancia del aporte del sistema informal de cuidados al sostenimiento de la salud. En ellos se afirma que sólo doce de cada cien horas

8 Delicado Useros, María. "Percepción de enfermería respecto a las cuidadores de personas dependientes", Libro de ponencias, VI Encuentro de Investigación en Enfermería, Investén-Isciii, Unidad de Coordinación y Desarrollo de Investigación en Enfermería, 20-23 de noviembre 2002, p. 135. 


\section{CRONICIDAD Y CUIDADORES FAMILIARES: UNA REVISIÓN DESDE LO CONTEXTUAL Y CONCEPTUAL}

dedicadas al cuidado de la salud se aplican desde el sistema institucional; el resto se efectúan en contextos no formales y por motivos no laborales ${ }^{9-10}$ Según un estudio sueco, el cuidador informal dedica una media de 299 horas al mes, ocho veces y media más que el sistema formal (Wimo et al., 2002) ${ }^{11}$.

Gran parte de las actividades que se realizan alrededor del cuidado del ser humano en situación de salud-enfermedad son efectuadas en escenarios cotidianos de la vida del ser humano a lo largo del ciclo vital, que hacen que exista una manera particular de percibir y valorar el cuidado, y esperar que éste reúna determinadas características o condiciones.

Este fenómeno de manera refleja se presenta en otros países (García Calvente, et al., 1993) ${ }^{12}$. En Canadá se estima que entre un 85 y un $90 \%$ del total de cuidados prestados es de tipo informal. En España diversas investigaciones confirman que la familia es la principal proveedora de cuidados de salud; del total de cuidados que reciben las personas mayores, entre el 80 y el $88 \%$ los recibe exclusivamente de la familia, mientras que los servicios formales proveen un 3\%. Un 20.7\% de los adultos presta ayuda para la realización de las actividades de la vida diaria (AVD) a una persona mayor con la que convive, y el $93.7 \%$ de ellos tienen vínculos familiares con la persona a la que cuida.

En Estados Unidos, las estadísticas aportan evidencia de que la familia y los amigos son las únicas personas que proporcionan cuidados en aproximadamente tres cuartas partes de los hogares. Un estudio reciente estimó que de cuatro hogares con teléfono, en uno había por lo menos un cuidador, esto se traducía en más de 22 millones de hogares en Estados Unidos donde se prestan cuidados. El $20 \%$ de éstos reportaron una condición relacionada con demencia ${ }^{13}$. En palabras De la Cuesta B. en el 2004, la poca cobertura de los sistemas sanitarios y sociales en los países en vías de desarrollo, como Colombia, hace pensar que en estos países la proporción bien puede ser mayor.

Cuando se habla de cuidado informal se habla del apoyo ofrecido por los miembros de la red familiar inmediata. Por lo general es una mujer, con relación de parentesco directa con el receptor de cuidado: hija, esposa y madre, ubicadas entre 45 y 65 años, sin actividad laboral distinta a la de ama de casa, con bajo nivel educativo, y de estratos inferiores de clase social ${ }^{14}$.

En particular en el contexto regional, diversos estudios ${ }^{15}$ 16-17-18-19 sobre el cuidado de los cuidadores familiares de adultos en situación crónica de enfermedad en

\footnotetext{
9 García Calvente, MM. El cuidado de la salud ¿Quién hace qué? Editorial Index de Enfermería, 1993, 6: 16-19.

10 Durán Ma. A. Un nuevo estatuto para el cuidado, Editorial Index de Enfermería, 1993; 6: 5.

11 Wimo, A., Strauss, E., Nordberg, G. Johansson L. Time spent on informal and formal care giving for persons with dementia in Sweden. Health Policy 2002; 61: 255-268.

12 IMSERSO. Cuidados en la vejez. El apoyo informal. Madrid: Ministerio de Asuntos Sociales, 1995 [consultado 23 diciembre 2006 ]. http://imserso-mayores.csic.es// estadísticas/información/informe

${ }^{13}$ De la Cuesta Benjumea, Carmen. Op. cit., p. 8.

${ }^{14}$ La Parra D. "Contribución de las mujeres y hogares más pobres a la producción de cuidados de salud informales". Gaceta Sanitaria, 2001, (15): 498-505.

${ }^{15}$ Vega Angarita, Olga Marina. "Habilidad del cuidado de los cuidadores primarios de adultos discapacitados por enfermedad cerebrovascular del Norte de Santander 2005". Trabajo de grado para obtener el título de magíster en enfermería con énfasis en el paciente crónico, Universidad Nacional de Colombia - Universidad Francisco de Paula Santander.

${ }^{16}$ González Escobar, Dianne Sofía. "Habilidad del cuidado de cuidadores familiares de personas en situación crónica de enfermedad por diabetes Mellitus 2005". Trabajo de grado para obtener el título de magíster en enfermería con énfasis en el paciente crónico, Universidad Nacional de Colombia -Universidad Francisco de Paula Santander.
} 


\section{CRONICIDAD Y CUIDADORES FAMILIARES: UNA REVISIÓN DESDE LO CONTEXTUAL Y CONCEPTUAL}

el Norte de Santander han podido establecer que la proporción de cuidadores familiares mujeres es casi cuatro veces mayor que la de los hombres. La mayoría de ellas ubicadas en la franja de edad que corresponde a la denominada generación intermedia, seguida de los cuidadores mayores de 60 años, con un bajo grado de escolaridad, con parentescos de consanguinidad como hijo, madre-padre, hermana, así como parentesco de filiación tales como esposa, nuera y cuñada, quienes ejercen el rol desde el mismo momento del diagnóstico y sin recibir ningún tipo de apoyo, con una dedicación al día que supera la siete horas.

Características sociodemográficas de los cuidadores informales como el género, parentesco, estado civil, ocupación y convivencia, se constituyen en las variables más importantes para predecir a qué persona o miembro del núcleo familiar le corresponde asumir de manera obligada este complejo rol. Según planteamientos de Barrera, en cada familia hay un cuidador que responde espontáneamente, o por necesidad, sin que se haya llegado a un acuerdo explícito entre las personas que componen la familia ${ }^{20}$.

Poco a poco, sin apenas darse cuenta de ello, la persona integra su nuevo rol de cuidadora en su vida diaria. Se conoce sobre algunas formas en las que el cuidador se inicia en su rol: por una enfermedad aguda y una hospitalización que requiere un período de convalecencia, tras un período de fragilidad asociado a un envejecimiento biológico normal que en forma progresiva exige más ayuda, y a causa de una enfermedad degenerativa de la que ya existían algunas evidencias.

Se han propuesto múltiples clasificaciones de los cuidadores. Según el cuidado brindado Ebersoles y Hess (1990) distinguen el anticipatorio, que comprende decisiones basadas en necesidades futuras del enfermo crónico, y anticipa necesidades inevitables que influyen en las elecciones hechas en el presente. El preventivo, que proporciona el cuidado alterando el ambiente y suministrando asistencia - servicio a la persona con enfermedad lo que impide su deterioro. El cuidado de supervisión, que brinda cuidado activo y de compromiso directo de naturaleza administrativa. El instrumental, que realiza el mantenimiento de actividades de la vida diaria en cuidados procedimentales. Finalmente, el protector que ampara de las consecuencias eventos que no pueden prevenirse, mantiene la autoestima de la persona enferma y previene la depresión frente a la inversión de roles y al deterioro físico y mental.

Según el nivel de responsabilidad del cuidador hacia el receptor de cuidado, los cuidadores se clasifican en cuidadores primarios y secundarios (Stone et al.). El cuidador primario o principal, es aquel que asume

\footnotetext{
17 Ramírez Ordóñez, Ma Mercedes. "Habilidad de cuidado de los cuidadores principales en el paciente con cáncer gastrointestinal 2005". Trabajo de grado para obtener el título de magíster en enfermería con énfasis en el paciente crónico. Universidad Nacional de Colombia - Universidad Francisco de Paula Santander.

18 Chávez C., Wilmer O., Estévez López, Erica P., Rueda B., Julieta. "Grado de preparación para el cuidado en casa de cuidadores familiares de adultos en situación de enfermedad crónica discapacitante neurovascular de la ESE Hospital Universitario Erasmo Meoz durante el período comprendido de marzo a julio de 2006". Trabajo de grado presentado como requisito para optar al título de enfermera, Universidad Francisco de Paula Santander.

${ }^{19}$ González E., Dianne Sofía, Vega Angarita, Olga Marina. "Estudio comparativo sobre la habilidad de cuidado entre cuidadores familiares de personas en situación de enfermedad crónica por enfermedad cerebrovascular y diabetes Mellitas en San José de Cúcuta, Norte de Santander".

20 Barrera Ortiz, Lucy. "Investigar en el cuidado del cuidador de personas que viven en situación de enfermedad crónica". En La investigación y el cuidado en América Latina, 1ª $^{a}$ ed., Unibiblos, 2005, Universidad Nacional de Colombia, p. 273.
} 


\section{CRONICIDAD Y CUIDADORES FAMILIARES: UNA REVISIÓN DESDE LO CONTEXTUAL Y CONCEPTUAL}

la total responsabilidad de la tarea, pasando por diferenciaciones según la ayuda formal o informal que reciben. El cuidador secundario, por el contrario, no ostenta la responsabilidad permanente de cuidado y su actuación es, en muchos casos, eventual.

Se han descrito ciertas razones por las que los cuidadores informales asumen esta tarea. Según Adame Portillo, en 1996, las personas que cuidan a sus familiares lo hacen por un deber moral, por altruismo, por reciprocidad, por gratitud y estima con la persona enferma, por sentimientos de culpa del pasado, y para obtener la aprobación social y familiar.

En el ámbito familiar la atención del cuidado informal es realizada principalmente por las mujeres como proveedoras de cuidado y de afecto, como legado generacional en razón de su naturaleza y por patrones culturales tradicionales socialmente asignados. Según Julve Negro (2004), la atribución de roles en función de género parece mantener su pauta más tradicional en lo que concierne a los cuidados y se considera correspondiente a la esfera del trabajo reproductivo. Estudios como los de Robinsón y Moen (1994), citados por Robles Silva ${ }^{21}$ en el 2003, sobre la probabilidad de que las mujeres sean cuidadoras en algún momento de su vida, concluyen que el rol de cuidador es, por una parte, un rol intermitente que se repite en varias etapas de su vida, pero también que un mínimo porcentaje de mujeres son cuidadoras en más de dos ocasiones. Afirman "que las mujeres que repiten en el rol de cuidadoras, pasan por varias etapas, en lo general correspondientes con los tres momentos demográficos de la vida de la mujer: la juventud, la adultez y la ancianidad. La repetición en este rol lleva a que las mujeres experimenten el cuidado como una carrera que está presente a lo largo de la vida, independientemente de a quién se cuide".

Históricamente este tipo de cuidado, según los planteamientos de Colliere ${ }^{22}$, es el más antiguo de la historia del mundo, vinculado tradicionalmente con lo connatural a la mujer, debido a la estrecha relación que tiene con la fecundidad y con todo aquello que contribuye con la manutención y desarrollo de la vida. Cuantitativamente representan el $60 \%$ de los cuidadores principales de personas mayores ${ }^{23}$, el $75 \%$ en el caso de personas con discapacidad ${ }^{24}$ y el $92 \%$ de los cuidadores de personas que necesitan atención en el hogar ${ }^{25}$

La literatura internacional y nacional documenta aspectos diferenciales en el tipo de atención brindada, cantidad de tiempo dedicado y percepción del cuidado según el género de los cuidadores. Al respecto, se indica que las mujeres dedican mayor tiempo al cuidado de los enfermos crónicos que los hombres. Realizan con mayor frecuencia actividades más pesadas y cotidianas, como son la realización de actividades de la vida diaria (AVD): a limentación, baño, eliminación, vestido, movilización y administración de

\footnotetext{
20 Barrera Ortiz, Lucy. "Investigar en el cuidado del cuidador de personas que viven en situación de enfermedad crónica". En La investigación y el cuidado en América Latina, 1ª. ed., Unibiblos, 2005, Universidad Nacional de Colombia, p. 273.

${ }^{21}$ Robles Silva, Leticia. "Una vida cuidando a los demás: una carrera de vida en ancianas cuidadoras". Libro-Ponencia presentado en el Simposio Viejos y Viejas, Participación Ciudadana e Inclusión Social y 51 Congreso Internacional de Americanistas, Santiago de Chile, 14-18 de julio de 2003, pp.1-4.

${ }^{22}$ Colliere, Françoise. Promover la vida, la práctica de las mujeres en virtud de los cuidados de enfermería, Lisboa. Sindicato de Enfermeras Portugueses, 1989.

23 IMSERSO. Cuidados en la vejez, Op. cit., p. 85

24 Jiménez A., Huele. La discapacidad en España: datos epidemiológicos. Madrid, Real Patronato sobre Discapacidad, 2002.

${ }^{25}$ García Calvente, MM., Mateo Rodríguez, Inmaculada, Eguiguren, Ana P. “El sistema informal de los cuidados en clave de desigualdad", Revista Gaceta Sanitaria, 2004; 18 (Supl. 1): 132-139.
} 


\section{CRONICIDAD Y CUIDADORES FAMILIARES: UNA REVISIÓN DESDE LO CONTEXTUAL Y CONCEPTUAL}

medicamentos; y actividades instrumentales de la vida diaria (AIVD): preparación de comida, limpieza de la ropa o casa y administración de las finanzas ${ }^{26}$

EL CUIDADO A LARGO PLAZO: Impacto en los cuidadores familiares.

El impacto de cuidar supone el incremento de la percepción de carga en los cuidadores informales, siendo más evidente en mujeres que en hombres y reflejada en problemas de salud, conflictos con familiares y amigos y restricción en el soporte social $^{27}$.

Diversos estudios (Bajo y Domínguez-Alcón, 1996; Abellán, 1997; Colectivo IOE, 1998; Paoletti, 1999; Yanguas, Leturia y Leturia, 2000; Roca y cols., 2000) reportan asociación entre los niveles altos de estrés y una serie de repercusiones en la salud física y psíquica de las cuidadoras, siendo los problemas cardiovasculares, osteoarticulares, pérdida del apetito, insomnio, nerviosismo, depresión y baja autoestima las manifestaciones más evidentes de este compromiso. Entre los estresores potenciales asociados al cuidado se han identificado el deterioro cognitivo y funcional y la duración de la enfermedad (Lotus Shyu, 2000).

Uno de los efectos que los cuidadores expresan con claridad se refiere a conflictos familiares por el desacuerdo entre la persona que cuida y los otros miembros del grupo en relación con el comportamiento, decisiones y actitudes de unos y otros hacia la persona mayor o por la forma en que se proporcionan los cuidados ${ }^{28}$. Procesos familiares como los conflictos intrafamiliares, el criticismo y la culpa son situaciones que pueden afectar la relación del cuidado y desempeño del rol.

Diversos autores afirman que el costo que asumen los cuidadores, en particular las mujeres, por el hecho de ser cuidadoras es elevado en términos de salud, calidad de vida, acceso al empleo y desarrollo profesional, relaciones sociales, disponibilidad del propio tiempo y repercusiones económicas ${ }^{29}$.

Si bien existe una cantidad significativa de referencias bibliográficas sobre los cuidadores en los países desarrollados, éstas son aún limitadas en países en desarrollo como Colombia. El conocimiento sobre la situación de los cuidadores familiares en el país y en la región es reducido, por lo cual la investigación efectuada al respecto se basa en las experiencias vividas en países desarrollados.

A nivel local algunos estudios revelan la situación en que se encuentran los cuidadores familiares de enfermos crónicos, sobre todo respecto a la habilidad para el cuidado, calidad de vida, sobrecarga del cuidador y preparación para el cuidado.

En cuanto a la habilidad de cuidado de los cuidadores familiares de personas en situación de enfermedad crónica ${ }^{30-31-32}$ se encontró en dos terceras partes de los cuidadores un nivel deficiente

\footnotetext{
${ }^{26}$ Zabagueli A., Juando C., Díaz M., Corrales, Cabrera E., Bover A., Sáenz de O. "El cuidado informal", Libro de ponencias, VIII Encuentro de Investigación en Enfermería, Investén Isciii, 17 al 20 noviembre 2004, Sevilla, p. 34.

${ }_{27}$ Barrera Ortiz, Lucy, Pinto Afanador, Natividad, Sánchez Herrera, Beatriz. "Habilidad de cuidado de cuidadores familiares de personas con enfermedad crónica: comparación de géneros". Revista Actualizaciones en Enfermería, 2006; 9(2): 9-143.

${ }_{28}$ Blanco, Lidia. "Habilidad de cuidado de los cuidadores de adultos en situación de enfermedad crónica discapacitante". Tesis para optar al título de maestría con énfasis en cuidado al paciente crónico", Bogotá, Universidad Nacional de Colombia, 2002.

29 García Calvente, MM., Mateo. Op.cit.

30 Vega Angarita, Olga Marina. Op. cit.

31 González Escobar, Dianne Sofía. Op. cit.

32 Ramírez Ordóñez, $M^{a}$. Mercedes. Op. cit.
} 


\section{CRONICIDAD Y CUIDADORES FAMILIARES: UNA REVISIÓN DESDE LO CONTEXTUAL Y CONCEPTUAL}

de habilidad para la entrega del cuidado, a pesar de cuidar desde el momento del diagnóstico, de tener un tiempo significativo en el ejercicio del cuidado y de la relación parental existente. Resultados que coinciden con los reportados por otras investigaciones efectuadas en el contexto colombiano y latinoamericano, que hacen evidente la necesidad de estrategias de apoyo dirigidas a esta población, que posibiliten el desarrollo de las habilidades necesarias para el desempeño del rol y la prestación de un cuidado de calidad a las personas en situación de enfermedad crónica.

En el análisis de la calidad de vida de los cuidadores familiares un estudio efectuado en el $2006^{33}$ identificó, cómo las acciones de cuidado que realizan los cuidadores tienen impacto significativo en sus vidas, en especial en el bienestar físico relacionado con fatiga, cambios en el apetito, alteraciones en el sueño; seguido del compromiso en el bienestar social, en aspectos como el aislamiento social, interferencia con el empleo, disminución del tiempo libre, situaciones que perturban, en forma notable, el equilibrio personal, familiar y laboral. La calidad de vida en el bienestar psicológico mostró su afectación con la presencia de ansiedad, desesperación y depresión por el rol en el que se encuentran y por la dificultad para lidiar con sus vidas, teniendo un familiar en situación de enfermedad crónica.
En relación con la sobrecarga en el cuidado ${ }^{34-35}$ se reporta que más de la mitad de los cuidadores familiares de personas en situación crónica de enfermedad perciben algún grado de sobrecarga en el cuidado. La sobrecarga a nivel físico se relaciona, en especial, con insuficiente tiempo para sí mismos, agobio por múltiples responsabilidades y deterioro de la salud desde el ejercicio del cuidado. La sobrecarga psíquica de los cuidadores se manifiesta por angustia, depresión, miedo a posibles complicaciones del familiar, sentimiento de abandono o falta de apoyo por parte de la familia.

Respecto al grado de preparación para el cuidado en casa de familiares adultos discapacitados un estudio $^{36}$ destaca que un porcentaje considerable de cuidadores no reúne los requisitos para asumir el rol en acciones particulares que demandan en los cuidadores conocimiento, habilidad para manejar la situación, persistencia, bienestar y desempeño.

Los cuidadores familiares experimentan situaciones ignoradas por el actual sistema de seguridad social, pues no cuenta con un servicio oportuno y eficiente para el fortalecimiento de la habilidad del cuidado en casa y el reconocimiento de la labor. Estos aspectos permiten la indagación de algunos referentes básicos para la enfermería, como la caracterización, la

\footnotetext{
33 Parra López, Erika Juliana. "Calidad de vida de los cuidadores familiares de adultos con enfermedad neurovascular discapacitante hospitalizados en el servicio de neurocirugía del Hospital Universitario Erasmo Meoz, San José de Cúcuta, durante los meses de octubre, noviembre y diciembre del 2006". Trabajo de grado presentado como requisito para optar al título de enfermera, Programa de Enfermería, Facultad de Ciencias de la Salud, Universidad Francisco de Paula Santander.

${ }^{34}$ Durán Delgado, Maritza; Mora Casariegos, Jhon James. "Valoración de la sobrecarga en el cuidador de pacientes diagnosticados de Alzheimer del Hospital Erasmo Meoz y la ESE Francisco de Paula Santander durante el primer semestre del 2004". Trabajo de grado para optar al título de enfermera, Programa de Enfermería, Facultad de Ciencias de la Salud, Universidad Francisco de Paula Santander, p. 62.

${ }^{35}$ Gallo Ferrizola, Elisa Johanna; Fuentes Yánez, Beatriz Adriana; Moros Farinango, Sandra Liliana. "Sobrecarga en el cuidador informal del niño(a) con discapacidad que asiste al programa de rehabilitación neurológica infantil de la ESE Centro de Rehabilitación Cardioneuromuscular de Norte de Santander durante el segundo semestre de 2006". Trabajo de grado para optar al título de enfermera, Programa de Enfermería, Facultad de Ciencias de la Salud, Universidad Francisco de Paula Santander, p. 54. ${ }^{36}$ Chávez Cañas, Wilmer; Estévez López, Erica P.; Rueda Barranco, Julieth J. "Grado de preparación para el cuidado en casa de cuidadores familiares de adultos en situación de enfermedad crónica discapacitante neurovascular de la ESE Hospital Universitario Erasmo Meoz durante el período comprendido entre marzo y julio de 2006". Trabajo de grado para optar al título de enfermera(o), Programa de Enfermería, Facultad de Ciencias de la Salud, Universidad Francisco de Paula Santander, p.
} 


\section{CRONICIDAD Y CUIDADORES FAMILIARES: UNA REVISIÓN DESDE LO CONTEXTUAL Y CONCEPTUAL}

importancia de su labor, las necesidades, la calidad de vida y la forma de vivir esa experiencia. Todos estos cambios plantean nuevos retos donde el cuidado de la salud y la vida toman sentido.

La Ley 266 de 1996 de enfermería reglamenta la profesión, y define el ámbito del ejercicio profesional. "El profesional de enfermería ejerce su práctica dentro de una dinámica interdisciplinaria, multiprofesional y transdisciplinaria, aporta al trabajo sectorial e intersectorial sus conocimientos y habilidades adquiridas en su formación universitaria y actualizada mediante la experiencia, la investigación, la educación continua. El profesional de enfermería ejerce sus funciones en los ámbitos donde la persona vive, trabaja, estudia, se recrea y se desarrolla, y en las instituciones que directa o indirectamente atienden la salud".

Hasta el momento en nuestro país y en la región los esfuerzos se han dirigido a brindar tratamiento al enfermo y se han olvidado del cuidador como persona clave en la asistencia, responsable del cuidado directo de aspectos de la vida del enfermo a quien cuidan, situación que puede resultar agobiante, limitar sus proyectos de vida y, en algunos casos, lo pueden llevar a sentir amenazado su bienestar físico o mental.

La enfermería como profesión dinámica que se fundamenta en el cuidado, ha observado estos cambios y en el país la Facultad de Enfermería de la Universidad Nacional de Colombia, en su Programa de Maestría en Enfermería, con el grupo de personas que conforman la sublínea de cuidado al paciente crónico, realiza una profundización sobre esta temática para identificar debilidades significativas relacionadas con la habilidad de cuidado y, por ende, dirige acciones a este grupo poblacional.

\section{REFERENTES BIBLIOGRÁFICOS}

1. OPS/OMS. La enfermería en la región de las Américas. Washington, 1999, p. 1.

2. ORGANIZACIÓN MUNDIAL DE LA SALUD. Prevención de las enfermedades crónicas una inversión vital. Nueva York: OMS, 2002, p. 23.

3. ORGANIZACIÓN MUNDIAL DE LA SALUD. Prevención de las enfermedades crónicas: una inversion vital: factores de riesgo. s. I: OMS, 2006, 5 pp.

4. DANE, "Proyecciones departamentales de población por sexo y edad", 1990-2015.

5. Ministerio de Salud y OPS. "Situación de salud en Colombia. Indicadores básicos 2001", Estándares (documento).

6. Boletín Epidemiológico del Norte de Santander, vol. 6 del 2005.

7. Estadística ESE Hospital Universitario Erasmo Meoz, 50 primeras causas de morbilidad sentida en el servicio de medicina interna, año 2005.

8. Delicado Useros, María. "Percepción de enfermería respecto a las cuidadores de personas dependientes", Libro de ponencias, VI Encuentro de Investigación en Enfermería, Investén-Isciii, Unidad de Coordinación y Desarrollo de Investigación en Enfermería, 20-23 de noviembre 2002, p. 135.

9. García Calvente, MM. El cuidado de la salud ¿Quién hace qué? Editorial Index de Enfermería, 1993, 6: 16-19. 


\section{CRONICIDAD Y CUIDADORES FAMILIARES: UNA REVISIÓN DESDE LO CONTEXTUAL Y CONCEPTUAL}

10. Durán Ma. A. Un nuevo estatuto para el cuidado, Editorial Index de Enfermería, 1993; 6: 5.

11. Wimo, A., Strauss, E., Nordberg, G. Johansson L. Time spent on informal and formal care giving for persons with dementia in Sweden. Health Policy 2002; 61: 255-268.

12. IMSERSO. Cuidados en la vejez. El apoyo informal. Madrid: Ministerio de Asuntos Sociales, 1995 [consultado 23 diciembre 2006]. http://imsersomayores.csic.es// estadísticas/información/informe

13. De la Cuesta Benjumea, Carmen. Op. cit., p. 8.

14. La Parra D. "Contribución de las mujeres y hogares más pobres a la producción de cuidados de salud informales". Gaceta Sanitaria, 2001, (15): 498-505.

15. Vega Angarita, Olga Marina. "Habilidad del cuidado de los cuidadores primarios de adultos discapacitados por enfermedad cerebrovascular del Norte de Santander 2005". Trabajo de grado para obtener el título de magíster en enfermería con énfasis en el paciente crónico, Universidad Nacional de Colombia - Universidad Francisco de Paula Santander.

16. González Escobar, Dianne Sofía. "Habilidad del cuidado de cuidadores familiares de personas en situación crónica de enfermedad por diabetes Mellitus 2005". Trabajo de grado para obtener el título de magíster en enfermería con énfasis en el paciente crónico, Universidad Nacional de Colombia - Universidad Francisco de Paula Santander.

17. Ramírez Ordóñez, $M^{a}$ Mercedes. "Habilidad de cuidado de los cuidadores principales en el paciente con cáncer gastrointestinal 2005". Trabajo de grado para obtener el título de magíster en enfermería con énfasis en el paciente crónico. Universidad Nacional 36 de Colombia - Universidad Francisco de Paula Santander.

18. Chávez C., Wilmer O., Estévez López, Erica P., Rueda B., Julieta. "Grado de preparación para el cuidado en casa de cuidadores familiares de adultos en situación de enfermedad crónica discapacitante neurovascular de la ESE Hospital Universitario Erasmo Meoz durante el período comprendido de marzo a julio de 2006". Trabajo de grado presentado como requisito para optar al título de enfermera, Universidad Francisco de Paula Santander.

19. González E., Dianne Sofía, Vega Angarita, Olga Marina. "Estudio comparativo sobre la habilidad de cuidado entre cuidadores familiares de personas en situación de enfermedad crónica por enfermedad cerebrovascular y diabetes Mellitus en San José de Cúcuta, Norte de Santander".

20. Barrera Ortiz, Lucy. "Investigar en el cuidado del cuidador de personas que viven en situación de enfermedad crónica". En La investigación y el cuidado en América Latina, $1^{a}$. ed., Unibiblos, 2005, Universidad Nacional de Colombia, p. 273.

21. Robles Silva, Leticia. "Una vida cuidando a los demás: una carrera de vida en ancianas cuidadoras". Libro-Ponencia presentado en el Simposio Viejos y Viejas, Participación Ciudadana e Inclusión Social y 51 Congreso Internacional de Americanistas, Santiago de Chile, 14-18 de julio de 2003, pp.1-4.

22. Colliere, Françoise. Promover la vida, la práctica de las mujeres en virtud de los cuidados de enfermería, Lisboa. Sindicato de Enfermeras Portugueses, 1989.

23. IMSERSO. Cuidados en la vejez, Op. cit., p. 85.

24. Jiménez A., Huele. La discapacidad en España: datos epidemiológicos. Madrid, Real Patronato sobre Discapacidad, 2002. 


\section{CRONICIDAD Y CUIDADORES FAMILIARES: UNA REVISIÓN DESDE LO CONTEXTUAL Y CONCEPTUAL}

25. García Calvente, MM., Mateo Rodríguez, Inmaculada, Eguiguren, Ana P. "El sistema informal de los cuidados en clave de desigualdad", Revista Gaceta Sanitaria, 2004; 18 (Supl. 1): 132-139.

26. Zabagueli A., Juando C., Díaz M., Corrales, Cabrera E., Bover A., Sáenz de O. "El cuidado informal", Libro de ponencias, VIII Encuentro de Investigación en Enfermería, Investén Isciii, 17 al 20 noviembre 2004, Sevilla, p. 34.

27. Barrera Ortiz, Lucy, Pinto Afanador, Natividad, Sánchez Herrera, Beatriz. "Habilidad de cuidado de cuidadores familiares de personas con enfermedad crónica: comparación de géneros". Revista Actualizaciones en Enfermería, 2006; 9(2): 9-143.

28. Blanco, Lidia. "Habilidad de cuidado de los cuidadores de adultos en situación de enfermedad crónica discapacitante". Tesis para optar al título de maestría con énfasis en cuidado al paciente crónico", Bogotá, Universidad Nacional de Colombia, 2002.

\section{García Calvente, MM., Mateo. Op.cit.}

30. Vega Angarita, Olga Marina. Op. cit.

31. González Escobar, Dianne Sofía. Op. cit.

32. Ramírez Ordóñez, Ma. Mercedes. Op. cit.

33. Parra López, Erika Juliana. "Calidad de vida de los cuidadores familiares de adultos con enfermedad neurovascular discapacitante hospitalizados en el servicio de neurocirugía del Hospital Universitario Erasmo Meoz, San José de Cúcuta, durante los meses de octubre, noviembre y diciembre del 2006". Trabajo de grado presentado como requisito para optar al título de enfermera, Programa de Enfermería, Facultad de Ciencias de la Salud, Universidad Francisco de Paula Santander.
34. Durán Delgado, Maritza; Mora Casariegos, Jhon James. "Valoración de la sobrecarga en el cuidador de pacientes diagnosticados de Alzheimer del Hospital Erasmo Meoz y la ESE Francisco de Paula Santander durante el primer semestre del 2004". Trabajo de grado para optar al título de enfermera, Programa de Enfermería, Facultad de Ciencias de la Salud, Universidad Francisco de Paula Santander, p. 62.

35. Gallo Ferrizola, Elisa Johanna; Fuentes Yánez, Beatriz Adriana; Moros Farinango, Sandra Liliana. "Sobrecarga en el cuidador informal del niño(a) con discapacidad que asiste al programa de rehabilitación neurológica infantil de la ESE Centro de Rehabilitación Cardioneuromuscular de Norte de Santander durante el segundo semestre de 2006". Trabajo de grado para optar al título de enfermera, Programa de Enfermería, Facultad de Ciencias de la Salud, Universidad Francisco de Paula Santander, p. 54.

36. Chávez Cañas, Wilmer; Estévez López, Erica P.; Rueda Barranco, Julieth J. "Grado de preparación para el cuidado en casa de cuidadores familiares de adultos en situación de enfermedad crónica discapacitante neurovascular de la ESE Hospital Universitario Erasmo Meoz durante el período comprendido entre marzo y julio de 2006". Trabajo de grado para optar al título de enfermera(o), Programa de Enfermería, Facultad de Ciencias de la Salud, Universidad Francisco de Paula Santander, p.

Fecha de recibido: Junio 20 de 2007

Fecha de aceptación: Diciembre 6 de 2007 